\title{
Disturbance of the OPG/RANK/RANKL pathway and systemic inflammation in COPD patients with emphysema and osteoporosis
}

Peng Bai ${ }^{1}$, Yongchang Sun ${ }^{1 *}$, Jianmin Jin ${ }^{1}$, Jia Hou ${ }^{1}$, Ran Li ${ }^{1}$, Qing Zhang ${ }^{2}$ and Yang Wang ${ }^{3}$

\begin{abstract}
Background: Osteoporosis is one of the systemic features of COPD. A correlation between the emphysema phenotype of COPD and reduced bone mineral density (BMD) is suggested by some studies, however, the mechanisms underlying this relationship are unclear. Experimental studies indicate that IL-1 $\beta, \mathrm{LL}-6$ and TNF- $\alpha$ may play important roles in the etiology of both osteoporosis and emphysema. The OPG/RANK/RANKL system is an important regulator of bone metabolism, and participates in the development of post-menopausal osteoporosis. Whether the OPG/RANK/RANKL pathway is involved in the pathogenesis of osteoporosis in COPD has not been studied.

Methods: Eighty male patients (current or former smokers) completed a chest CT scan, pulmonary function test, dual x-ray absorptiometry measurements and questionnaires. Among these subjects, thirty patients with normal BMD and thirty patients with low BMD were selected randomly for measurement of IL-1 $1 \beta$, IL-6, TNF- $\alpha$ (flow cytometry) and OPG/RANK/RANKL (ELISA). Twenty age-matched healthy volunteers were recruited as controls.
\end{abstract}

Results: Among these eighty patients, thirty-six had normal BMD and forty-four had low BMD. Age, BMI and CAT score showed significant differences between these two COPD groups $(p<0.05)$. The low-attenuation area (LAA\%) in the lungs of COPD patients was negatively correlated with lumbar vertebral BMD $(r=0.741 ; p<0.0001)$. Forward logistic regression analysis showed that only LAA\% $(p=0.005)$ and BMI $(p=0.009)$ were selected as explanatory variables. The level of IL-1 $\beta$ was significantly higher in the COPD patients as compared to the normal controls $(p<0.05)$, but the difference between the two COPD groups did not reach significance. The levels of IL- 6 and TNF- $\alpha$ among the three groups were significantly different $(p<0.05)$. The level of RANKL and the RANKL/OPG ratio were significantly higher in COPD patients with low BMD compared to those with normal BMD and the normal controls $(p<0.05)$, and correlated negatively with lumbar vertebral BMD, but positively with LAA\%.

Conclusions: Radiographic emphysema is correlated with low BMD in current and former smokers with COPD. IL$1 \beta, I L-6, T N F-\alpha$, and the osteoporosis-related protein system OPG/RANK/RANKL may have some synergetic effects on emphysema and bone loss in COPD.

Keywords: chronic obstructive pulmonary disease, pulmonary emphysema, osteoporosis, cytokine, OPG/RANK RANKL

\section{Background}

Chronic obstructive pulmonary disease (COPD) is recognized as a highly prevalent condition which causes significant morbidity and mortality [1], and commonly associated with many extra-pulmonary abnormalities

\footnotetext{
* Correspondence: suny@ccmu.edu.cn

'Department of Respiratory Medicine, Beijing Tongren Hospital, Capital

Medical University, Beijing, China

Full list of author information is available at the end of the article
}

such as cardiovascular disease, cachexia, skeletal muscle wasting, and anemia [2,3]. Osteoporosis is one of the most important systemic comorbidities in COPD, which increases the risk of osteoporotic fractures, and carries a heavy economic burden [4]. It has been reported that bone mineral density (BMD) is lower in COPD patients than in healthy subjects [5-7]. Studies have shown that low BMD in COPD patients is related to some clinical and physiological indices, such as lung function $\left(\mathrm{FEV}_{1}\right)$,

\section{Biomed Central}


low body weight and decreased fat-free mass [8,9]. BMD can be measured by Dual X-ray Absorptiometry (DXA). With the advantages of high precision, short scan times, and low radiation dose, DXA provides a noninvasive method of diagnosing osteoporosis and guiding decisions about treatment $[10,11]$.

Emphysema is a primary imaging manifestation in COPD patients, and has been recognized as an important phenotype of COPD. The low-attenuation area (LAA) in the lungs evaluated by chest CT images has been widely used to quantitatively assess pulmonary emphysema [12]. However, the association between emphysema and osteoporosis in COPD patients and its possible underlying mechanism are still unclear. In fact, similarities between parenchymal emphysema and osteoporosis, including the loss of extracellular matrix and common association with inflammatory mediators, alludes to a potential mechanistic link between the two processes.

Patients with COPD have evidence of systemic inflammation, which may be responsible for some comorbidities. Levels of inflammatory cytokines such as IL-1 $\beta$, IL6 and TNF- $\alpha$ are increased in the systemic circulation of COPD patients [13-15]. Inflammatory cytokines, including IL-1 $\beta$, IL- 6 and TNF- $\alpha$, are also responsible for the characteristic loss of bone density in osteoporosis through their effect on osteoclast activity $[16,17]$. The osteoporosis related-protein triad osteoprotegerin (OPG)/receptor activator of NF- $\kappa \mathrm{B}$ (RANK)/RANK ligand (RANKL) has been identified as an important regulator of bone metabolism and remodeling. This system has been shown to interact with IL-1 $\beta$, IL- 6 and TNF- $\alpha$, and play important roles in the occurrence of postmenopausal osteoporosis $[18,19]$. This raises the intriguing possibility that the OPG/RANK/RANKL pathway may also be involved in the development of osteoporosis in COPD.

Our hypothesis was that the extent of emphysema was associated with the severity of osteoporosis in COPD patients, and that pro-inflammatory cytokines and the OPG/RANK/RANKL system were altered and correlated with osteoporosis in these patients.

\section{Methods}

Subjects

Eighty male COPD patients diagnosed as clinically stable were enrolled from January 2010 to May 2011. Female patients were excluded from the study to avoid the influence of postmenopausal osteoporosis. COPD was diagnosed according to the criteria of the Global Initiative for Chronic Obstructive Lung Disease (GOLD) [2]. All patients were more than 40 years old, and were current or former smokers. The exclusion criteria were as follows: (1) acute exacerbation of COPD in the last 3 months, (2) history of respiratory diseases other than COPD, (3) history of chest surgery, (4) occurrence of malignancy within the previous five years, (5) history of bone disease, (6) current and/or previous inhale, oral or intravenous corticosteroid therapy, (7) other systemic diseases, such as renal insufficiency and thyroid diseases, or medication which may influence bone metabolism within the previous one year.

The study was approved by the local ethics committee of Beijing Tongren Hospital, Capital Medical University (TRECKT 2008-14). All subjects provided informed consent to join the study.

On the recruiting day, each subject completed a chest CT scan, prebronchodilator and post-bronchodilator spirometry and plethysmography, and DXA measurements of BMD of the femoral neck and lumbar spine. Recording of demographic parameters and medical history, evaluation of quality of life, and collection of blood samples were also completed on that day.

\section{Analysis of LAA}

CT scans were performed using a multidetector row scanner (Brilliance 64, Phillips, Eindhoven, Netherlands) with technical parameters of $120 \mathrm{kVp}$, slice thicknesses of $1.0 \mathrm{~mm}$, and a thread interval of $11.08 \mathrm{~mm}$. During scanning, subjects were asked to hold their breath after a deep inspiration in the supine position.

The percentage of LAA (LAA\%) was measured automatically using specified software in the Extended Brilliance Workspace to quantitatively evaluate pulmonary emphysema. All CT images from the upper margin of the aortic arch to the diaphragm were used to calculate the LAA\%. Lung fields were defined as areas with a CT scan density of less than -200 Hounsfield units (HU), and the threshold between the LAA and normal lung density was defined as -950 HU. The LAA\% was calculated as (number of LAA pixels in all slices)/(total lung area) $\times 100(\%)$. The measurements of LAA\% were performed independently by an experienced radiologist, who was blinded to the subjects' clinical information.

\section{Measurements of BMD}

BMD at the lumbar spine (L1-L4) and the bilateral femoral neck was measured by Dual X-Ray Absorptiometry (Lunar prodigy, GE Healthcare, London, United Kingdom). BMD is reported as an absolute value (g/ $\mathrm{cm}^{2}$ ) and a $\mathrm{T}$ score, which represents the number of standard deviations from a young, sex- and ethnic group-specific reference mean. According to the definition of the World Health Organization (WHO), T scores are used as the basis for diagnosis as follows: normal bone mineral density: $T$ score greater than -1 at both sites (femoral neck and lumbar spine), osteopenia: $\mathrm{T}$ score less than or equal to -1 but greater than -2.5 at 
either site, osteoporosis: $\mathrm{T}$ score less than or equal to -2.5 at either site [20]. On the basis of this standard, we classified the subjects into two groups: COPD with normal BMD ( $\mathrm{T}$ score greater than -1 at both sites) (36 patients) and COPD with low BMD (osteopenia/osteoporosis, $\mathrm{T}$ score less than or equal to -1 at either site) (44 patients).

\section{Measurements of IL-1 $\beta$, IL-6, TNF- $\alpha$ and OPG/RANK/ RANKL}

Among the eighty subjects, thirty patients with normal BMD and thirty patients with low BMD were randomly selected for the measurement of serum cytokines. Twenty healthy volunteers (current or former smokers, mean age: $58.4 \pm 7.1$ ), whose BMD at the lumbar spine (L1-L4) and the bilateral femoral neck measured by DXA were normal, were recruited as normal controls. The smoking index showed no significant difference among these three groups $(p>0.05)$. The exclusion criteria were the same as those used for the COPD subjects. Fasting blood samples (approximately $20 \mathrm{~mL}$ ) were collected by venipuncture in plain tubes. Sera were obtained by centrifugation at $1000 \times \mathrm{g}$ for $5 \mathrm{~min}$ at room temperature. The samples were stored at $-80^{\circ} \mathrm{C}$ until analysis. The levels of IL-1 $\beta$, IL- 6 , and TNF- $\alpha$ in serum samples were evaluated by flow cytometry (CBA Kit, BD Bioscience, USA). OPG/RANK/RANKL concentrations were measured by ELISA (Catalog No.CSBE04692h, 14931h, 08157h; Cusabio Biotech Co., Ltd, USA). Assays were performed according to the manufacturer's protocol.

\section{Evaluation of Clinical Characteristics}

Dyspnea was rated using the American Thoracic Society MMRC dyspnea score [21].

Six-Minute Walking Distance (6MWD): The patients walked in the same hospital hallway without oxygen supplementation encouraged by a technician. Processing was consistent with the ATS guidelines for the six minute walk test [22].

BODE indices were calculated as the sum score proposed by Celli et al. [23], based on the following four variables: (1) $\mathrm{FEV}_{1}$ percentage of predicted ( $\geq 65 \%$, score $0 ; 50 \%$ to $64 \%$, score $1 ; 36 \%$ to $49 \%$, score 2 ; and $\leq 35 \%$, score 3); (2) Six-minute walking distance (6MWD) ( $\geq$ $350 \mathrm{~m}$, score $0 ; 250$ to $349 \mathrm{~m}$, score $1 ; 150$ to $249 \mathrm{~m}$, score 2; $\leq 149 \mathrm{~m}$, score 3 ); (3) MMRC dyspnea score (0 to 1 , score $0 ; 2$, score $1 ; 3$, score 2 ; and 4 , score 3$)$; and (4) body mass index (BMI) (> $21 \mathrm{~kg} / \mathrm{m}^{2}$, score $0 ; \leq 21 \mathrm{~kg} / \mathrm{m}^{2}$, score 1). Possible total scores ranged from 0 to 10 .

All patients were assessed by interview using the COPD Assessment Test (CAT) [24], and the scores were recorded.

\section{Data analysis}

All statistical analyses were performed using a statistical software package (Statistics Package for the Social Sciences, SPSS 17.0, Inc., Chicago, IL, USA). Data are expressed as the mean \pm SD. Comparisons of data between two groups (COPD with normal BMD and COPD with low BMD) were performed by $\mathrm{T}$-test (for normal distribution parameters) and Mann-Whitney $U$ test (for abnormal distribution parameters). Since the subjects' age among control, COPD with normal BMD and COPD with low BMD groups had significant difference $(p<0.05)$, the comparisons of serum cytokines were performed by analysis of covariance. The relationships among continuous parameters were evaluated by the multiple linear regression analyses. Based on the grouping of BMD, forward logistic regression analyses were performed to evaluate the contribution of LAA\% and other parameters which could cause a significant difference between these two groups. A p value of < 0.05 was considered significant.

\section{Results}

Demographic parameters, pulmonary function results and quality of life score of eighty COPD patients

Comparisons of age, $\mathrm{BMI}, \mathrm{FEV}_{1} / \mathrm{FVC}, \mathrm{FEV}_{1} \%$ predicted, CAT score and BODE index between the two COPD groups (COPD with normal BMD and COPD with low BMD) are shown in Table 1. Age, BMI and CAT score showed significant differences between these two groups, but there was no significant difference in $\mathrm{FEV}_{1} /$ FVC, $\mathrm{FEV}_{1} \%$ predicted, smoking index and BODE index.

\section{CT scan measurements for pulmonary emphysema}

As a quantitative assessment of pulmonary emphysema, the mean value of LAA\% for all 80 COPD patients was $24.66 \pm 12.90 \%$. Values of LAA\% were $18.67 \pm 10.00 \%$ and $29.57 \pm 13.03 \%$ for COPD with normal and low BMD groups, respectively, with a significant difference between groups $(p<0.0001)$.

\section{BMD in the femoral neck and lumbar spine}

To reduce the influence of BMD variability among vertebral levels and between bilateral femoral necks, for each patient, the mean BMD of four lumbar vertebrae and of both femoral necks were used as the final results. The mean BMD of the femoral neck showed a good correlation with that of the lumbar vertebrae $(r=0.959$; $p<0.0001$ ), a measurement which has been widely used for the assessment of BMD (Figure 1). Therefore, we used lumbar vertebral BMD to further analyze the correlation of BMD with anthropometric parameters, quality of life score, and LAA\%. 
Table 1 Comparison of demographic parameters, pulmonary function results and Quality of Life Score between two COPD groups

\begin{tabular}{llll}
\hline Variables & COPD with normal BMD $(\mathbf{n}=\mathbf{3 6})$ & COPD with low BMD $(\mathbf{n}=\mathbf{4 4})$ & p-value \\
\hline Age $(\mathrm{yr})$ & $65.5 \pm 5.1$ & $67.8 \pm 5.2$ & 0.049 \\
BMI $\left(\mathrm{kg} / \mathrm{m}^{2}\right)$ & $26.1 \pm 3.6$ & $23.5 \pm 2.9$ & $<0.001$ \\
Smoking index(pack-yr) & $28.1 \pm 26.0$ & $26.7 \pm 21.6$ & 0.79 \\
CAT score & $15.8 \pm 9.6$ & $21.0 \pm 8.5$ & 0.01 \\
BODE index & $3.3 \pm 1.1$ & $4.1 \pm 1.2$ & 0.26 \\
FEV 1 FVC(\%) & $57.0 \pm 10.1$ & $53.9 \pm 9.6$ & 0.17 \\
FEV 1 \% predicted(\%) & $58.4 \pm 19.2$ & $52.8 \pm 17.3$ & 0.18 \\
\hline
\end{tabular}

Data are mean (SD) unless otherwise stated.

COPD: chronic obstructive pulmonary disease, BMD: bone mineral density, BMI: body mass index, CAT: COPD Assessment Test, BODE index: body mass index, airflow obstruction, dyspnea, and exercise capacity index, FEV1: forced expiratory volume in one second

\section{Serum level of pro-inflammatory cytokines and OPG/} RANK/RANKL

Serum levels of IL- $1 \beta$, IL-6, TNF- $\alpha$ and OPG/RANK/ RANKL were compared among the three groups (normal control, COPD with normal BMD and COPD with low BMD) (Table 2). For adjustment of the effect of age, analysis of covariance was used (age as the covariance).

The level of IL-1 $\beta$ was significantly higher in the COPD patients as compared to the normal controls $(p<$ $0.05)$, but the difference between the two COPD groups did not reach significance. The levels of IL- 6 and TNF$\alpha$ among the three groups were significantly different ( $p$ $<0.05$ ) (Figure 2). No significant difference existed in the level of OPG and RANK among the three groups, but the level of RANKL (Figure 3) and the ratio of RANKL/OPG (R/O) (Figure 4) were remarkably higher in COPD patients with low BMD compared to those with normal BMD and the normal controls $(p<0.05)$.

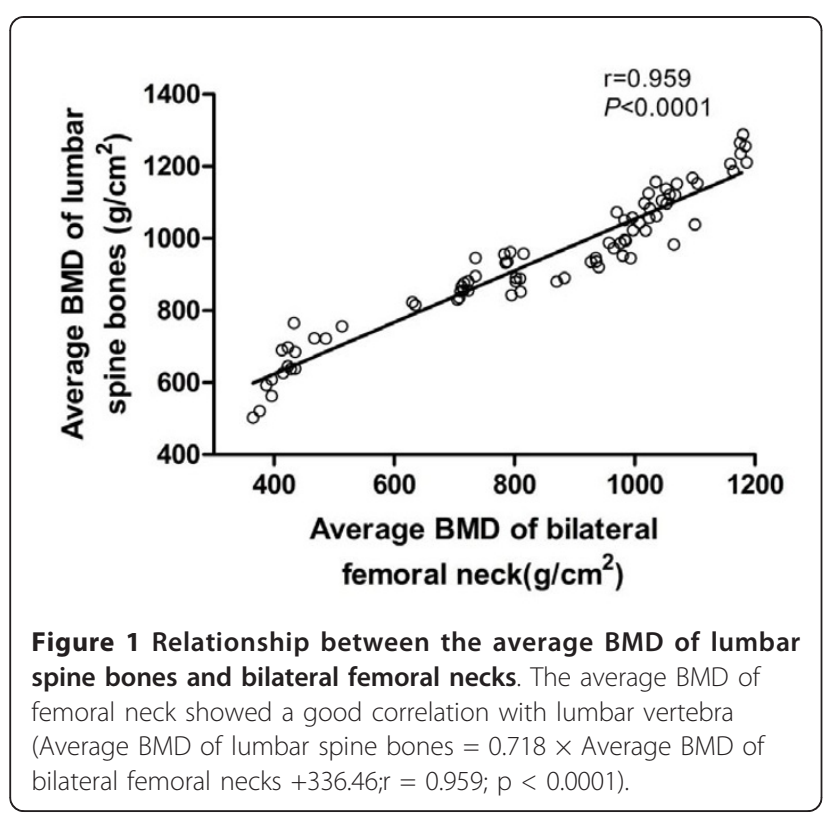

Correlation of BMD with pro-inflammatory cytokines and OPG/RANK/RANKL concentration

Multiple linear regression analyses showed that, with the adjustment of age, among IL-1 $\beta$, IL-6, TNF- $\alpha$, OPG, RANK, RANKL and the ratio of RANKL/OPG, only the level of RANKL $(r=-0.256, p=0.015)$ and the ratio of RANKL/OPG ( $r=-0.258, p=0.015)$ had a significant negative correlation with lumbar vertebral BMD.

\section{Correlation of pulmonary emphysema severity (LAA\%)} with proinflammatory cytokines and OPG/RANK/RANKL concentration

Multiple linear regression analyses showed that, with the adjustment of age, among IL- $1 \beta$, IL- 6 , TNF- $\alpha$, OPG, RANK, RANKL and the ratio of RANKL/OPG, only the level of RANKL $(r=0.216, p=0.043)$ and the ratio of RANKL/OPG ( $\mathrm{r}=0.213, p=0.041)$ had a significant positive correlation with LAA\%.

\section{Correlation of BMD with pulmonary emphysema severity (LAA\%) and other variables}

Pearson product-moment correlation analyses revealed that LAA\% had a significant negative correlation with lumbar vertebral BMD ( $\mathrm{r}=-0.741 ; p<0.0001)$. Because the complex relationship between COPD and osteoporosis is confounded by the overlap of common risk factors, a forward logistic regression analysis was performed, based on the grouping of BMD and factors which showed a significant difference between the two COPD groups (i.e., LAA\%, age, BMI and CAT) as independent variables. This showed that only LAA\% $(p=0.005)$ and BMI $(p=0.009)$ were explanatory variables, indicating that LAA\% is the strongest predictor of osteopenia/ osteoporosis.

\section{Discussion}

COPD has been recognized as a systemic disease which also involves several extra-pulmonary features. Osteoporosis is one of the major systemic comorbidities of COPD, which is more prevalent among COPD patients 
Table 2 Comparison of IL-1 $\beta$,IL-6, TNF- $\alpha$ and OPG/RANK/RANKL levels among three groups

\begin{tabular}{llll}
\hline Variables & normal control $(\mathbf{n}=\mathbf{2 0})$ & COPD with normal BMD $(\mathbf{n}=\mathbf{3 0})$ & COPD with low BMD $(\mathbf{n}=\mathbf{3 0})$ \\
\hline $\mathrm{IL}-1 \beta(\mathrm{fg} / \mathrm{ml})$ & $122.21 \pm 35.14$ & $186.83 \pm 104.87 \dagger$ & $192.94 \pm 115.82 \dagger$ \\
$\mathrm{IL}-6(\mathrm{fg} / \mathrm{ml})$ & $354.37 \pm 83.14$ & $421.52 \pm 125.78 \dagger$ & $483.73 \pm 110.38 \dagger \neq$ \\
$\mathrm{TNF}-\alpha(\mathrm{fg} / \mathrm{ml})$ & $193.09 \pm 81.20$ & $261.28 \pm 96.09 \dagger$ & $324.37 \pm 141.29 \dagger \neq$ \\
$\mathrm{OPG}(\mathrm{pg} / \mathrm{ml})$ & $236.50 \pm 38.76$ & $240.43 \pm 65.77$ & $257.97 \pm 86.80$ \\
$\mathrm{RANK}(\mathrm{pg} / \mathrm{ml})$ & $82.28 \pm 29.76$ & $73.90 \pm 50.41$ & $2.24 \pm 0.80 \dagger \neq$ \\
\hline
\end{tabular}

Data are mean (SD) unless otherwise stated.

$\mathrm{tp}<0.05$ compared with the normal control group.

$\neq \mathrm{p}<0.05$ compared with the COPD with normal BMD group

COPD: chronic obstructive pulmonary disease, BMD: bone mineral density, IL: interleukin, TNF- $\alpha$ :tumor necrosis factor $\alpha$, OPG: osteoprotegerin, RANK: receptor activator of NF- $\kappa$ B, RANKL: RANK ligand

than healthy subjects $[5,8]$. The etiology of COPDrelated osteoporosis is almost certainly multifactorial. With the exception of BMI [6-9], the effects of other factors which have been reported to be responsible for the reduced bone density in patients with COPD, such as age [6], poor quality of life [6], $\mathrm{FEV}_{1}[6,9]$, smoking [6], and corticosteroid therapy $[6,8,25]$ remain controversial. In our study, BMI was lower in the COPD with low BMD group than the COPD with normal BMD group, and logistic regression analysis showed that BMI was an independent predictor for BMD. This result is in accordance with previous studies [6-9]. In our study, patients in both of the two COPD groups were older than the control, and the COPD patients with low BMD were older than those with normal BMD, the difference among these three groups being significant $(p<0.05)$. But age was not an independent variable in logistic regression analysis. The effect of age on COPD-related bone loss is still indefinite, and age may influence the serum level of pro-inflammatory cytokines. Therefore, when performing the data analysis, we used analysis of

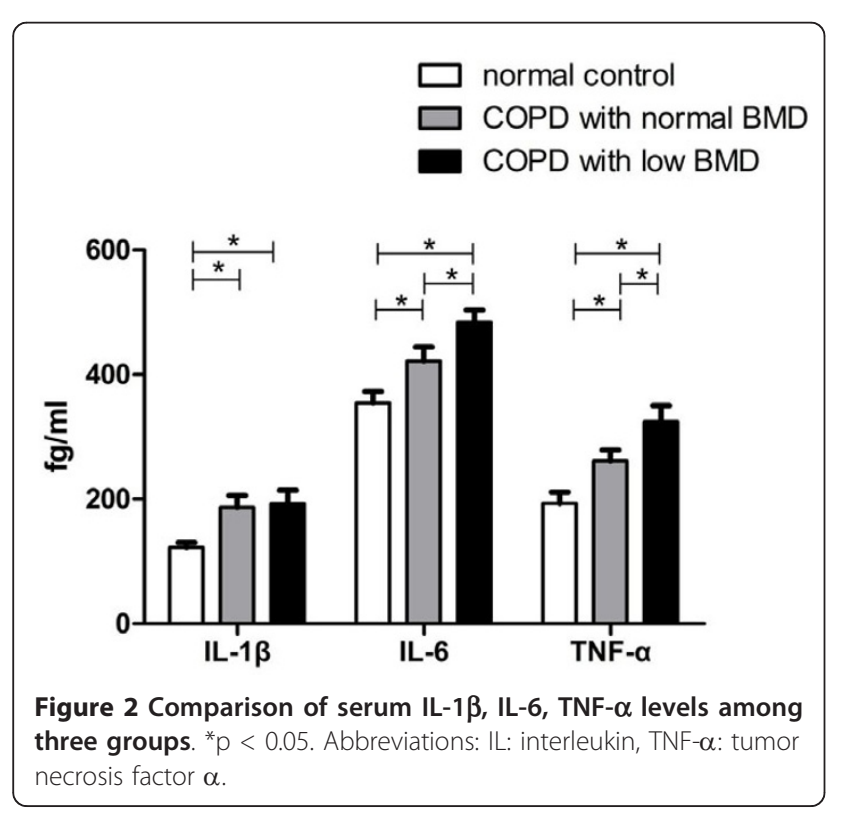

covariance and multiple linear regression to correct the influence of age. BMI and age are both correlated with quality of life. CAT score and BODE index are two new indices used to evaluate the quality of life for COPD patients. The trend in our study showed that patients with lower BMD had higher CAT scores (a relationship which was statistically significant) and BODE index (which did not reach statistical significance), indicating a deterioration in the quality of life. This result agrees with the findings of Iqbal et al. [6], showing that BMD correlated weakly with activity evaluated by St George's respiratory questionnaire (SGRQ), whereas Katsura and Kida [7] and Kjensli et al. [8] did not find a significant correlation with activity evaluated by the 6MWD. Several studies have found no significant relationships between bone density and airflow obstruction $[7,26]$, and these are in agreement with our study: $\mathrm{FEV}_{1} / \mathrm{FVC}$ and $\mathrm{FEV}_{1} \%$ predicted showed no significant difference between the two COPD groups. In the study of Duckers et al., over $80 \%$ of the male COPD with osteopenia/

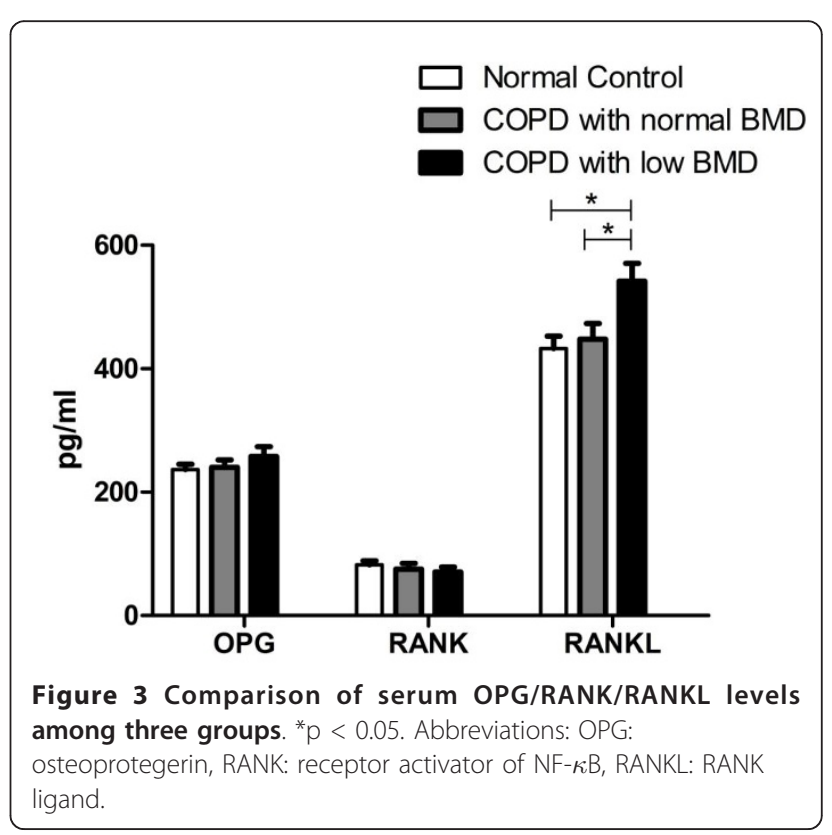




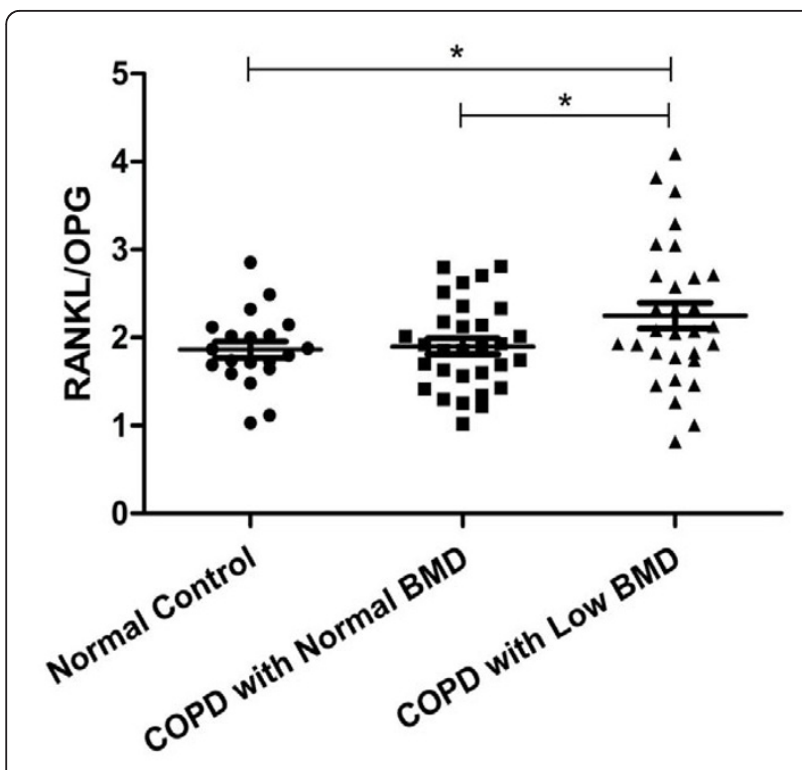

Figure 4 Comparison of RANKL/OPG among three groups. ${ }^{*} \mathrm{p}<$ 0.05. Abbreviations: OPG: osteoprotegerin, RANKL: receptor activator of $\mathrm{NF}-\kappa \mathrm{B}$ ligand.

osteoporosis had mild to moderate severity airways obstruction(GOLD I and II) [27]. Further studies are needed to investigate the relationships between bone density and airflow obstruction.

In the present study, we demonstrated that LAA\%, which reflects the extent of emphysema, correlated significantly with reduced bone density. In addition, logistic regression analysis showed that only LAA\% and BMI could predict bone density in male COPD patients. It means that, in addition to the effect of BMI, LAA\% is also an independent predictor of bone loss and responsible for the difference which we has found between COPD patients with low BMD and those with normal BMD. This result is in accordance with that of Ohara and coworkers, who found a linear correlation between emphysema and absolute BMD of the thoracic and L1 vertebrae measured by quantitative CT [28]. Recently, a study by Bon and coworkers also showed that radiographic emphysema was a strong, independent predictor of low BMD in current and former smokers with COPD [20]. These results provide support for the hypothesis that emphysema itself could be a risk factor for bone loss.

Destruction of the lung parenchyma may be associated in some way with the mechanism of the destruction of bone structure. The underlying mechanisms are speculative and may be related to systemic inflammatory factors acting synchronously on the lung parenchyma and bone matrix. IL-1 $\beta$, IL- 6 and TNF- $\alpha$ are all well-established inflammatory cytokines involved in the systemic inflammation of COPD. In our study, the serum levels of IL-
$1 \beta$, IL- 6 and TNF- $\alpha$ in COPD patients were all significantly higher than in controls, supporting the existence of systemic inflammation in COPD. Bon and coworkers have demonstrated an association between distinct serum inflammatory mediators and quantitative CT measurements of emphysema [29]. Simultaneously, IL$1 \beta$, IL- 6 and TNF- $\alpha$ have been shown to be important modulators of bone metabolism and remodeling, by stimulating osteoclast differentiation in a synergistic fashion [16]. These cytokines may not only regulate osteoclastogenesis via their effects on stromal cells, but also act directly on osteoclasts and their precursors [30]. In addition, they also interact with the osteoporosisrelated OPG/RANK/RANKL protein system [18].

Recently, the OPG/RANK/RANKL system has been shown to have pleiotropic effects on both bone metabolism [31] and the immune system [32], and has led to a new molecular perspective on osteoclast biology and bone homeostasis. RANKL and OPG, synthesized by stromal cells/osteoblasts, have been identified as two principal cytokines controlling osteoclastic differentiation and activation. RANK, localized at the cell surface of mature osteoclasts and osteoclastic precursors, is the third protagonist $[33,34]$. The predominant role of RANKL in bone physiology is the stimulation of osteoclastic differentiation/activation and the inhibition of osteoclast apoptosis [35]. Binding of RANKL to RANK stimulates (1) differentiation of osteoclastic precursors into mature osteoclasts, and (2) activation of mature osteoclasts. OPG acts as a decoy receptor for RANKL and thus down-regulates RANKL signaling through RANK. It represents an endogenous decoy receptor that neutralizes the biological effects of all forms of RANKL and thus acts as an inhibitor of bone resorption [33,34]. In fact, bone remodeling appears to be mainly controlled by the balance of RANKL/OPG. Any modification in the RANKL-to-OPG ratio can induce either excessive bone resorption or, in contrast, excessive bone formation [35]. Dysregulation of their relative expression can lead to pathological conditions such as osteopenia/ osteoporosis, bone tumor-associated osteolysis, immune disease, or cardiovascular pathology [16]. It has been demonstrated both in vivo and in vitro, that IL-1 $\beta$, IL-6 and TNF- $\alpha$, which are the products of stromal cells and monocytes, increase production of both RANKL and OPG. The dominant outcome of these three cytokines is a net increase in RANKL activity, which leads to bone loss. In addition, increases of RANKL and OPG can also up-regulate the expression of IL- 6 and TNF- $\alpha$ [19], which may enhance the systemic inflammation of COPD.

In the present study, we found that the serum levels of IL- 6 , TNF- $\alpha$, RANKL and the ratio of RANKL/OPG were significantly higher in the COPD with low BMD 
group than in the other two groups (controls and normal BMD); the level of IL-1 $\beta$ was also higher in the low BMD group than in the normal BMD group, but did not reach statistical significance. Only the level of RANKL and the ratio of RANKL/OPG were significantly correlated with lumbar vertebral BMD (negative) and LAA\% (positive).

It is well known that smoking can not only influence the serum level of inflammatory cytokines (included IL$1 \beta$, IL-6, TNF- $\alpha$ and OPG/RANK/RANKL system), but also accelerate the bone loss. In our study, patients and the control were all current or former smokers, and the smoking index showed no significant difference among these three groups $(p>0.05)$. But further studies involving both smoking and non-smoking subjects are needed.

In the study of Duckers et al., the serum level of OPG was greater in COPD patients who combined with osteopenia/osteoporosis than those without low BMD and related inversely to hip BMD, but the level of RANKL and the ratio of RANKL/OPG were not determined [27]. In our study, the level of OPG did not significantly differ among these three groups. However, the study of Eagan and coworkers found that the level of OPG was significantly lower in COPD patients [36]. Both our study and that of Eagan et al. showed that, in COPD patients, the balance of the OPG/RANK/RANKL system is destroyed and manifests a dominant trend for RANKL. Thus, we assume that systemic inflammation may increase the serum levels of inflammatory cytokines (especially, IL- 6 and TNF- $\alpha$ ), which then disturb the balance of the OPG/RANK/RANKL system and result in a dominant trend for RANKL. This trend may cause the bone loss and osteopenia/osteoporosis seen among COPD patients. Further studies will be necessary to validate our hypothesis.

There are several limitations to our study. Firstly, the subjects were all male. Female patients, who have been reported to have a much higher incidence of osteopenia/osteoporosis, were not involved. Second, this study did not include patients who never smoked. Third, the size of the study population was relatively small. Further studies, involving more patients, including both males and females, as well as smoking and non-smoking subjects, are necessary.

\section{Conclusions}

In conclusion, our study demonstrated that the extent of pulmonary emphysema was significantly correlated with reduced bone density, and for the first time to our knowledge, that the level of RANKL and the RANKL/OPG ratio were significantly higher in COPD patients with low BMD compared to those with normal BMD, and correlated negatively with lumbar vertebral BMD, but positively with LAA\%. Although osteoporosis results from multiple causes such as age, smoking, malnutrition, and steroid treatment, the systemic inflammation of COPD may contribute directly to osteoporosis to some extent. There may be congenerous molecular mechanisms involved in the destruction of both lung parenchyma and bone structure, and inflammatory cytokines, such as IL-1 $\beta$, IL- 6 and TNF$\alpha$, as well as the osteoporosis related protein triad OPG/RANK/RANKL, may have some synergetic effects on both emphysema and bone loss. Further studies are needed to explore the mechanisms underlying COPDrelated bone loss.

\section{Abbreviations}

COPD: chronic obstructive pulmonary disease; BMD: bone mineral density; DXA: Dual X-ray Absorptiometry; LAA: low-attenuation area; OPG: osteoprotegerin; RANK: receptor activator of NF-KB; RANKL: RANK ligand; $\mathrm{FEV}_{1}$ : forced expiratory volume in one second; FVC: forced vital capacity; BMI: body mass index; 6MWD: six-minute walking distance; CAT: COPD Assessment Test; GOLD: the Global Initiative for Chronic Obstructive Lung Disease.

\section{Acknowledgements}

Funding/Support

This work was supported by Beijing Natural Science Foundation (No. 7092022).

The authors thank Husheng Zhang, Yang Wang, Xiufang Luo, Haiyang Sheng and Shanna Xu for their support on screening participating subjects throughout the study. Thanks for the help of data analysis from Binrong Ma. All authors read and approved the final manuscript.

\section{Author details}

'Department of Respiratory Medicine, Beijing Tongren Hospital, Capital Medical University, Beijing, China. ²Department of Radiology, Beijing Tongren Hospital, Capital Medical University, Beijing, China. ${ }^{3}$ Central Laboratory, Beijing Tongren Hospital, Capital Medical University, Beijing, China.

\section{Authors' contributions}

PB completed the recruiting of patients, performed the collection and analysis of all data and blood samples, and was a major contributor in writing the manuscript.

YS was the primary investigator of this study and was a major contributor in writing the manuscript.

$\mathrm{JJ}$ and $\mathrm{JH}$ were two major contributors in writing the manuscript

$\mathrm{RL}$ completed the recruiting of patients, performed the collection of clinical data.

QZ performed the evaluation of low-attenuation area (LAA) in the lungs on chest CT images

YW performed the laboratory-based assays.

All authors read and approved the final manuscript.

\section{Competing interests}

The authors declare that they have no competing interests.

Received: 10 October 2011 Accepted: 16 December 2011 Published: 16 December 2011

\section{References}

1. Lopez AD, Shibuya K, Rao C, Mathers CD, Hansell AL, Held LS, Schmid V, Buist S: Chronic obstructive pulmonary disease: current burden and future projections. Eur Respir J 2006, 27:397-412.

2. Rabe KF, Hurd S, Anzueto A, Barnes PJ, Buist SA, Calverley P, Fukuchi Y, Jenkins C, Rodriguez-Roisin R, Van Weel C, Zielinski J: Global strategy for the diagnosis, management, and prevention of chronic obstructive 
pulmonary disease: Gold executive summary. Am J Respir Crit Care Med 2007, 176:532-555.

3. Agusti AG, Soriano JB: COPD as a systemic disease. COPD 2008, 5:133-138.

4. National Osteoporosis Foundation. Physician's guide to prevention and treatment of osteoporosis. Washington, DC. National Osteoporosis Foundation; 2003.

5. Agusti AG, Noguera A, Sauleda J, Sala E, Pons J, Busquets X: Systemic effects of chronic obstructive pulmonary disease. Eur Respir J 2003, 21:347-360

6. Iqbal F, Michaelson J, Thaler L, Rubin J, Roman J, Mark S: Declining bone mass in men with chronic pulmonary disease: contribution of glucocorticoid treatment, body mass index, and gonadal function. Chest 1999, 116:1616-1624.

7. Katsura H, Kida K: A comparison of bone mineral density in elderly female patients with COPD and bronchial asthma. Chest 2002, 122:1949-1955.

8. Kjensli A, Mowinckel P, Ryg MS, Falch JA: Low bone mineral density is related to severity of chronic obstructive pulmonary disease. Bone 2007, 40:493-497.

9. Vrieze A, de Greef MH, Wy'kstra PJ, Wempe JB: Low bone mineral density in COPD patients related to worse lung function, low weight and decreased fat-free mass. Osteoporos Int 2007, 18:1197-1202.

10. Mineo TC, Ambrogi V, Mineo D, Fabbri A, Fabbrini E, Massoud R: Bone mineral density improvement after lung volume reduction surgery for severe emphysema. Chest 2005, 127:1960-1966.

11. Blake GM, Knapp KM, Fogelman I: Dual X-ray absorptiometry-clinical evaluation of a new cone-beam system. Calcif Tissue Int 2005, 76:113-120.

12. Madani A, Zanen J, de Maertelaer V, Gevenois PA: Pulmonary emphysema: objective quantification at multi-detector row $\mathrm{CT}$; comparison with macroscopic and microscopic morphometry. Radiology 2006, 238:1036-1043.

13. Garcia-Rio F, Miravitlles M, Soriano JB, Muñoz L, Duran-Tauleria E, Sánchez G, Sobradillo V, Ancochea J, EPI-SCAN Steering Committee: Systemic inflammation in chronic obstructive pulmonary disease: a population-based study. Respiratory Research 2010, 11:63.

14. Barnes PJ, Celli BR: Systemic manifestation and comorbidities of COPD. Eur Respir J 2009, 33:1165-1185.

15. Hurst JR, Perera WR, Wilkinson TM, Donaldson GC, Wedzicha JA: Systemic and upper and lower airway inflammation at exacerbation of chronic obstructive pulmonary disease. Am J Respir Crit Care Med 2006, 173:71-78.

16. Gregory R, Mundy MD: Osteoporosis and Inflammation. Nutrition Reviews 2007, 65:S147-S151.

17. McLean RR: Proinflammatory cytokines and osteoporosis. Curr Osteoporos Rep 2009, 7:134-139.

18. Kwan TS, Padrines M, Théoleyre S, Heymann D, Fortun Y: IL-6, RANKL, TNFalpha/LL-1: interrelations in bone resorption pathophysiology. Cytokine Growth Factor Rev 2004, 15:49-60

19. Théoleyre S, Wittrant Y, Tat SK, Fortun Y, Redini F, Heymann D: The molecular triad OPG/RANK/RANKL: involvement in the orchestration of pathophysiological bone remodeling. Cytokine Growth Factor Rev 2004, 15:457-475.

20. Bon J, Fuhrman CR, Weissfeld JL, Duncan SR, Branch RA, Chang CH, Zhang Y, Leader JK, Gur D, Greenspan SL, Sciurba FC: Radiographic Emphysema Predicts Low Bone Mineral Density in a Tobacco-exposed Cohort. Am J Respir Crit Care Med 2011, 183:885-890.

21. American Thoracic Society: Surveillance for respiratory hazards in the occupational setting. Am Rev Respir Dis 1982, 126:952-956.

22. ATS Committee on Proficiency Standards for Clinical Pulmonary Function Laboratories: ATS statement: guidelines for the six minute walk test. Am J Respir Crit Care Med 2002, 116:111-117.

23. Celli BR, Cote CG, Marin JM, Casanova C, Montes de Oca M, Mendez RA, Plata P: The body-mass index, airflow obstruction, dyspnea, and exercise capacity index in chronic obstructive pulmonary disease. $N$ Engl J Med 2004, 350:1005-1012.

24. Jones PW, Harding G, Berry P, Wiklund I, Chen WH, Leidy NK: Development and first validation of the COPD Assessment Test. Eur Respir J 2009, 34:648-654.

25. Lee TA, Weiss KB: Fracture risk associated with inhaled corticosteroid use in chronic obstructive pulmonary disease. Am J Respir Crit Care Med 2004, 169:855-859.
26. Bolton CE, lonescu AA, Shiels KM, Pettit RJ, Edwards PH, Stone MD, Nixon LS, Evans WD, Griffiths TL, Shale DJ: Associated loss of fat-free mass and bone mineral density in chronic obstructive pulmonary disease. Am J Respir Crit Care Med 2004, 170:1286-1293.

27. Duckers JM, Evans BA, Fraser WD, Stone MD, Bolton CE, Shale DJ: Low bone mineral density in men with chronic obstructive pulmonary disease. Respiratory Research 2011, 12:101.

28. Ohara T, Hirai T, Muro S, Haruna A, Terada K, Kinose D, Marumo S, Ogawa E, Hoshino Y, Niimi A, Chin K, Mishima M: Relationship between pulmonary emphysema and osteoporosis assessed by $\mathrm{CT}$ in patients with COPD. Chest 2008, 134:1244-1249.

29. Bon JM, Leader JK, Weissfeld JL, Coxson HO, Zheng B, Branch RA, Kondragunta V, Lee JS, Zhang Y, Choi AMK, Lokshin AE, Kaminski N, Gur D, Sciurba FC: The influence of radiographic phenotype and smoking status on peripheral blood biomarker patterns in chronic obstructive pulmonary disease. PLOS ONE 4(8):e6865.

30. Ragab AA, Nalepka JL, Bi Y, Greenfield EM: Cytokines synergistically induce osteoclast differentiation: support by immortalized or normal calvarial cells. Am J Physiol Cell Physiol 2002, 283:C679-687.

31. Horowitz MC, Xi Y, Wilson K, Kacena MA: Control of osteoclastogenesis and bone resorption by members of the TNF family of receptors and ligands. Cytokine Growth Factor Rev 2001, 12:9-18.

32. Fouque-Aubert $A$, Chapurlat R: Influence of RANKL inhibition on immune system in the treatment of bone diseases. Joint Bone Spine 2008, 75:5-10.

33. Simonet WS, Lacey DL, Dunstan CR, Kelley MC, Hang MS, Luthy R, Nguyen HQ, Wooden S, Bennett L, Boone T, Shimamoto G, DeRose M, Elliott R, Colombero A, Tan HL, Trail G, Sullivan J, Davy E, Bucay N, RenshawGegg L, Hughes TM, Hill D, Pattison W, Campbell P, Boyle WJ: Osteoprotegerin: a novel secreted protein involved in the regulation of bone density. Cell 1997, 89:309-319.

34. Lacey DL, Timms E, Tan HL, Kelley MJ, Dunstan CR, Burgess T, Elliott R, Colombero A, Elliott G, Scully S, Hsu H, Sullivan J, Hawkins N, Davy E, Capparelli C, Eli A, Qian YX, Kaufman S, Sarosi I, Shalhoub V, Senaldi G, Guo J, Delaney J, Boyle WJ: Osteoprotegerin ligand is a cytokine that regulates osteoclast differentiation and activation. Cell 1998, 93:165-176.

35. Khosla S: Minireview: the OPG/RANKL/RANK system. Endocrinology 2001, 142:5050-5055

36. Eagan $T$, Ueland T, Wagner PD, Hardie JA, Mollnes TE, Dams JK, Aukrust $P$, Bakke PS: Systemic inflammatory markers in COPD: results from the Bergen COPD Cohort Study. Eur Respir J 2010, 35:540-548.

doi:10.1186/1465-9921-12-157

Cite this article as: Bai et al:: Disturbance of the OPG/RANK/RANKL pathway and systemic inflammation in COPD patients with emphysema and osteoporosis. Respiratory Research 2011 12:157.

\section{Submit your next manuscript to BioMed Central and take full advantage of:}

- Convenient online submission

- Thorough peer review

- No space constraints or color figure charges

- Immediate publication on acceptance

- Inclusion in PubMed, CAS, Scopus and Google Scholar

- Research which is freely available for redistribution

Submit your manuscript at www.biomedcentral.com/submit
Ciomed Central 